\title{
Assistência de enfermagem a um paciente com anemia falciforme em unidade de terapia intensiva
}

\author{
Nursing care in sickle cell anemia in an intensive care unit
}

Atención de enfermería en anemia falciforme en una unidad de cuidados intensivos

Juliette Nobre dos Santos Silva ${ }^{1 *}$, Wanne Thaynara Vaz Gurjão ${ }^{1}$, Josué Rodrigues de Sousa ${ }^{1}$, Jonas Melo de Matos Junior ${ }^{1}$, Rosilene da Silva ${ }^{1}$, Suellen Patricia Sales da Costa Loureiro ${ }^{1}$, Danielle Saraiva Tuma dos Reis ${ }^{1}$, Karina de Oliveira Freitas ${ }^{1}$, Cláudia Ribeiro Menezes ${ }^{1}$, Esleane Vilela Vasconcelos ${ }^{1}$.

\begin{abstract}
RESUMO
Objetivo: Relatar a construção do processo de enfermagem, por meio da Sistematização da assistência de enfermagem elaborada por enfermeiros residentes de uma unidade de terapia intensiva, a um paciente com anemia falciforme e artrite séptica, desde sua admissão a alta da unidade. Relato de experiência: Para a realização do presente estudo utilizou-se a Sistematização da assistência de enfermagem para a elaboração dos cuidados de enfermagem, por meio iniciando pela coleta de dados, seguida do exame físico cefalopodal. A partir da evolução, definiu-se os problemas potenciais e reais que conduziram na definição de seis diagnósticos de enfermagem como risco de aspiração, risco de choque, risco de infecção, volume de líquidos excessivos, dor aguda e troca de gases prejudicada e suas respectivas intervenções, a fim de alcançar a resolução dos problemas elencados. Considerações finais: Ao paciente com hemoglobinopatia a implementação do processo de Enfermagem fundamentou a tomada de decisão na resolução da instabilidade hemodinâmica, melhora da oxigenação, conforto e redução de riscos relacionados a assistência à saúde.
\end{abstract}

Palavras-chave: Anemia falciforme, Processo de enfermagem, Unidades de terapia intensiva.

\begin{abstract}
Objective: To report the construction of the nursing process, through the systematization of nursing care developed by nurses resident in an intensive care unit, to a patient with sickle cell anemia and septic arthritis, from admission to discharge from the unit. Experience report: In order to carry out the present study, Nursing Care Systematization was used for the elaboration of nursing care, starting with data collection, followed by a cephalopodal physical examination. From the evolution, the potential and real problems that led to the definition of six nursing diagnoses were defined as risk of aspiration, risk of shock, risk of infection, volume of excessive fluids, acute pain and impaired gas exchange and their respective interventions in order to achieve the resolution of the listed problems. Final considerations: To the patient with hemoglobinopathy, the implementation of the Nursing process was the basis for decision making in the resolution of hemodynamic instability, improvement of oxygenation, comfort and reduction of risks related to health care.
\end{abstract}

Key words: Sickle cell anemia, Nursing process, Intensive care units.

\section{RESUMEN}

Objetivo: Informar la construcción del proceso de enfermería, a través de la sistematización de los cuidados de enfermería desarrollados por enfermeras residentes en una unidad de cuidados intensivos, a un paciente con anemia falciforme y artritis séptica, desde el ingreso hasta el alta de la unidad. Informe de experiencia:

${ }^{1}$ Universidade Federal do Pará (UFPA), Belém - PA. *E-mail: juliettenobre@hotmail.com

SUBMETIDO EM: 1/2021

ACEITO EM: 2/2021

PUBLICADO EM: 22021 
Para la realización del presente estudio se utilizó la Sistematización del Cuidado de Enfermería para la elaboración del cuidado de enfermería, comenzando con la recolección de datos, seguida de un examen físico cefalópodo. A partir de la evolución, los problemas potenciales y reales que llevaron a la definición de seis diagnósticos de enfermería se definieron como riesgo de aspiración, riesgo de shock, riesgo de infección, volumen de líquidos excesivos, dolor agudo y alteración del intercambio gaseoso y sus respectivas intervenciones para lograr la resolución de los problemas enumerados. Consideraciones finales: Para el paciente con hemoglobinopatía, la implementación del proceso de Enfermería fue la base para la toma de decisiones en la resolución de la inestabilidad hemodinámica, mejora de la oxigenación, comodidad y reducción de riesgos relacionados con el cuidado de la salud.

Palabras clave: Anemia falciforme, Proceso de enfermería, Unidades de cuidados intensivos.

\section{INTRODUÇÃO}

A anemia falciforme (AF) é uma patologia de etiologia genética, com prevalência em populações de origem africana. A mesma é determinada pela mutação do cromossomo 11, onde se tem a substituição de um ácido glutâmico por uma valina na cadeia beta globina, que dá origem a hemoglobina S. Por sua configuração diferente, os eritrócitos assumem um formato de foice, em decorrência da polimerização da hemoglobina em condições de hipoxemia (COTTLE RN, et al., 2016).

Geralmente a sintomatologia clínica tem origem ainda nos primeiros meses de vida e se manifestam em decorrência da hemólise e da oclusão vascular. Os portadores apresentam como manifestação aguda da doença, dor, surdez, anemia aguda, priaprismo e infarto pulmonar. Ao longo do tempo e com a evolução da doença surgem as manifestações crônicas, resultante de alterações em diversos órgãos e sistemas, como infecções recorrentes, complicações pulmonares, renais, neurológicas, hepatobiliares e oculares entre outras (HABARA A, et al., 2016).

$\mathrm{Na} A \mathrm{~F}$ a agressão esplênica reduz a capacidade de funcionamento do baço resultando na diminuição da capacidade fagocítica e produção de imunoglobulinas, que predispõe o indivíduo a processos infecciosos de etiologia bacteriana, como por exemplo, a artrite séptica, aumentando potencialmente o risco para sepse e por conseguinte a morte se não diagnosticada e tratada na fase inicial (GUEDES A, et al., 2010).

A artrite séptica é ocasionada por meio da disseminação hematogênica ou por solução de contiguidade, podendo também está relacionada à iatrogenias (GUEDES A, et al., 2010). É uma doença frequente em portadores de AF e se manifesta como infecção do líquido sinovial, piogênica, no interior das articulações, acometendo, em sua maioria, indivíduos na idade pediátrica, com incidência de 12 casos para cada 100.000 habitantes/ano (KENNEDY N, et al., 2015; ASSUNÇÃO JH, et al., 2018). Até o momento não há dados específicos do Brasil sobre a incidência desta infecção osteoarticular.

Durante a exacerbação da doença, o paciente poderá necessitar de cuidados de maior complexidade, como por exemplo de uma unidade de terapia intensiva (UTI). Destaca-se que a UTI possui como objetivo a recuperação e suporte para manutenção das funções vitais do indivíduo, sendo este um ambiente de alta tecnologia e com profissionais especializados, que devem desenvolver suas habilidades de acordo com a tecnologia disponível, buscando sempre oferecer um cuidado individualizado, humanizado e holístico (RODRIGO L, 2018).

$\mathrm{O}$ indivíduo portador de AF atendido em unidades de alta complexidade, requerem um atendimento imediato em decorrência de sua fragilidade secundária as crises álgicas e episódios infecciosos graves, que acabam por repercutir não somente em sua qualidade de vida, mas que também colocam em risco o seu estado hemodinâmico (SOARES EPB, et al., 2014). Dentre as complicações que requerem internação em UTI destaca-se a: dor, anemia, acidente vascular cerebral e quadro séptico (ADEGBOLA MA, 2011).

Portanto, entende-se que o cuidado prestado a esse perfil clínico exige certo conhecimento sobre questões hematológicas e imunológicas, assim como uma avaliação individualizada, planejamento baseado nas 
necessidades individuais e um olhar reflexivo para a tomada de decisão, de forma que se favoreça o fornecimento de uma prática assistencial adequada e livre de danos, resultando em benefícios para o paciente (ROCHA LPO, et al., 2014). Dentro deste contexto tem-se a Sistematização da Assistência de Enfermagem (SAE) que é uma ferramenta fundamental para o planejamento do cuidado no ambiente hospitalar, tanto em aspectos relativos à prevenção e autocuidado, quanto aos curativos.

Assistência que de acordo com Carvalho EMMS (2014) em sua revisão integrativa é pouco divulgada nas produções científicas no contexto da Enfermagem. Assim, com o intuito de colaborar na redução destas lacunas, o presente estudo objetivou relatar a construção do processo de enfermagem, por meio da Sistematização da assistência de enfermagem elaborada por enfermeiros residentes de uma unidade de terapia intensiva, a um paciente com anemia falciforme e artrite séptica, desde sua admissão a alta da unidade.

\section{RELATO DE EXPERIÊNCIA}

Os enfermeiros residentes, atuantes na Unidade de Terapia Intensiva (UTI) de um hospital público referência no Estado do Pará em doenças infectoparasitárias, resolveram aplicar o conhecimento acerca do Processo de Enfermagem a um caso de Anemia Falciforme com complicação para artrite séptica, em um adolescente internado, durante as suas vivências práticas neste setor, através da elaboração dos diagnósticos de Enfermagem pertinentes ao caso clínico e segundo a taxonomia Nursing Diagnostic Terminology (NANDA), contribuindo para a oferta de um cuidado mais individualizado, eficaz e atenda a todas as necessidades do paciente.

Trata-se de um paciente do sexo masculino, dezessete anos, com diagnóstico de anemia falciforme, acompanhado desde a infância, que permaneceu internado por cinco dias em uma UTI com crise de calcificação, insuficiência respiratória aguda hipoxêmica anêmica e sepse de foco articular. Segundo registro em prontuário o menor apresentou trauma em joelho direito dias antes a internação, no quarto dia após o trauma, evoluiu com algia em joelho direito, aumento de volume local e calor, com dificuldade de extensão do membro mais claudicação. Procurou assistência médica em unidade de pronto atendimento, onde recebeu tratamento sintomático. Evoluindo posteriormente com dor torácica, hipertermia, cefaleia e dispneia intensa com necessidade de oxigenoterapia por cateter nasal.

$\mathrm{Na}$ admissão atual paciente torporoso, GLASGOW 9, edemaciado, em uso de macronebulização com 10 I/min, Sat O2 de 86\%, respiração tipo Kussmaul, ictérico, edema de membro inferior direito (MID) com flexão prejudicada e dor à mobilização, pulso presente, acesso periférico salinizado em membro superior direito (MSD), diurese em uropen. Paciente normocárdico, hipertérmico $\left(38,9^{\circ} \mathrm{C}\right)$, taquipneico, normotenso, som pulmonar limpo, ausculta cardíaca sem sopros, hepatomegalia, e esplenomegalia importantes. Exames laboratoriais evidenciando anemia (Hemoglobina $(\mathrm{Hb}): 2,5 \mathrm{~g} / \mathrm{dl}$; Hematócrito $(\mathrm{Ht}$ ): 7,6), uréia: 48mg/dl, creatinina: 0,2 mg/dl, Transaminase Glutâmico-oxalacética (TGO): 1065, Transaminase Glutâmico-pirúvica (TGP): 325, leucócitos 34.120/mm3, e gasometria demonstrando acidose metabólica compensada (PH: 7,42; PCO2: 31; HCO3: 20; Sat O2: 100). Iniciado antibioticoterapia com oxacilina e cefepime. E administrado concentrado de hemácia e analgesia.

Para a elaboração e implementação do processo de Enfermagem, foram elencados os problemas potenciais e ativos, a fim de assegurar a eleição dos diagnósticos pertinentes e resolutivos ao caso. Os problemas potenciais selecionados foram o rebaixamento de nível de consciência, sepse, realização de procedimentos invasivos. A partir destes, definiu-se como diagnósticos de enfermagem (DE) o risco de aspiração, risco de choque e risco de infecção respectivamente.

Já os problemas ativos foram definidos como edema, dor, hepatoesplenomegalia, hipoxemia, elegendose como DE, volume de líquidos excessivos, dor aguda e troca de gases prejudicada. Destaca-se que todos os diagnósticos de Enfermagem e suas respectivas intervenções, basearam-se na taxonomia NANDA e Nursing Interventions Classification (NIC) e nas melhoras evidências científicas a respeito da temática (Quadro 1). 
Quadro 1 - Diagnósticos e Prescrições de enfermagem adaptados para o caso.

\begin{tabular}{|c|c|}
\hline Diagnósticos de Enfermagem & Prescrições de Enfermagem \\
\hline Risco de aspiração & $\begin{array}{l}\text { - Monitorar o nível de consciência, o reflexo de tosse, reflexo } \\
\text { do vômito e capacidade de deglutir. } \\
\text { - Monitorar a condição pulmonar. } \\
\text { - Elevar cabeceira à } 45^{\circ} \text { graus } \\
\text { - Instalar cânula de guedel } \\
\text { - Alimentar o paciente em pequenas quantidades. }\end{array}$ \\
\hline Risco de Choque & $\begin{array}{l}\text { - Monitorizar sinais vitais } 2 / 2 \text { hs } \\
\text {-Usar monitoramento por linha arterial para melhorar a } \\
\text { precisão dos dados da pressão sanguínea } \\
\text { - Monitorar balanço Hídrico } 2 / 2 \text { hs } \\
\text {-Avaliar nível de consciência } \\
\text {-Administrar oxigenoterapia conforme prescrição médica. } \\
\text { - Administrar expansores de volume para manter os } \\
\text { parâmetros hemodinâmicos, conforme prescrito. }\end{array}$ \\
\hline Risco de infecção & $\begin{array}{l}\text { - Lavagem das mãos antes e após realização de procedimentos } \\
\text { ou após contato com paciente e superfícies próximas ao paciente. } \\
\text { - Realizar troca de cateter periférico a cada } 72 \text { a } 96 \text { hs. } \\
\text { - Realizar troca de fixação de AVP quando necessário (registrar } \\
\text { data e hora do curativo). } \\
\text { - Monitorar o surgimento de sinais de flebite (p. ex., dor, } \\
\text { hiperemia, calor na pele, edema) do AVP M/T/N. } \\
\text { - Inserir o cateter AVP usando técnica asséptica. } \\
\text { - Lavar o cateter com solução adequada após cada uso. }\end{array}$ \\
\hline Volume de Líquidos excessivos & $\begin{array}{l}\text { - Monitorar balanço Hídrico } 2 / 2 \text { hs } \\
\text { - Monitorar ingestão e eliminação M/T/N } \\
\text { - Monitorar resultados laboratoriais relevantes à retenção de } \\
\text { líquidos (p. ex., gravidade específica aumentada, níveis de ureia } \\
\text { aumentados, de hematócritos diminuídos e aumento da } \\
\text { osmolalidade urinária). } \\
\text { - Ajustar uma taxa de fluxo apropriado para infusão } \\
\text { intravenosa (ou transfusão de sangue). } \\
\text { - Monitorar os líquidos EV prescritos quanto à adequação (p. } \\
\text { ex., evitar água à vontade em paciente com sobrecarga hídrica e } \\
\text { hiponatremia). } \\
\text { - Administrar os diuréticos prescritos conforme apropriado. } \\
\text { - Monitorar mudanças no peso do paciente. }\end{array}$ \\
\hline Dor aguda & $\begin{array}{l}\text { - Anotar localização, tipo, frequência, intensidade e gravidade } \\
\text { da dor (3 vezes ao dia, de acordo com a escala de face álgica); } \\
\text { - Administrar analgésico conforme prescrição médica } \\
\text { - Monitorar SSVV 2/2 hs }\end{array}$ \\
\hline Troca de gases prejudicada & $\begin{array}{l}\text { - Manter cabeceira elevada a } 30 \text { à } 45 \text { graus } \\
\text { - Realizar controle de SSVV } \\
\text { - Realizar avaliação neurológica } \\
\text { - Monitoramento da gasometria }\end{array}$ \\
\hline
\end{tabular}

Fonte: Silva JNS, et al., 2020, baseado no North American Nursing Diagnosis Association (2012-2014).

Com base nos conhecimentos acerca da Sistematização da Assistência de Enfermagem, realizou-se o planejamento dos cuidados partindo dos diagnósticos e prescrições, tais cuidados foram implementados e checados diariamente pela equipe de técnicos de Enfermagem e enfermeiros. Em virtude de grande queixa álgica e risco de choque séptico distributivo, foram priorizados os cuidados como a oxigenoterapia, analgesia para o controle da dor, expansão volêmica com líquidos isotônicos prescritos para evitar hipotensão.

O planejamento foi diário e ajustado conforme os problemas encontrados a partir da evolução de enfermagem realizada três vezes ao dia, em cada turno, e conforme anotações sobre mudanças no estado clínico e das discussões com a equipe multiprofissional. 
Após a implementação dos cuidados o paciente apresentou importante melhora do quadro álgico, aumento da Sat O2 para 97\% após oferta de oxigênio suplementar no primeiro dia de internação, sendo suspenso após melhora da taquipneia e adequada perfusão tecidual, na ocasião foi instituída antibioticoterapia em virtude da leucocitose e administrado $240 \mathrm{ml}$ de concentrado de hemácia, com normalização dos níveis de hemoglobina. No terceiro dia paciente apresentou constipação intestinal, pela redução da motilidade gástrica ao uso de analgésico opióide, para o qual foi administrado óleo mineral com boa resposta á terapêutica.

O mesmo recebeu alta da UTI no quinto dia, apresentando-se em bom estado geral, hemodinamicamente estável, sem dor, eupneico e com leucócitose em remissão, com capacidade de acompanhamento do caso em enfermaria.

\section{DISCUSSÃO}

A artrite séptica (AS) é mais frequente nas articulações do joelho e quadril, tendo como principal agente etiológico, o Staphylococcus aureus, sendo, portanto, o principal alvo da terapia empírica precoce, tendo em consideração que o tempo sem tratamento pode comprometer cada vez mais a articulação do paciente, podendo levar à destruição da cartilagem e/ou à perda da função articular normal (SOARES CBG, et al., 2019).

Antes do diagnóstica estima-se que cerca de $20 \%$ dos pacientes apresentem algum histórico de trauma local inespecífico nas últimas duas semanas que antecedem o diagnóstico, como contusões, quedas e escoriações. Entende-se que o dano articular ocasionada pela AS pode aumentar mesmo após a erradicação dos microrganismos, dado a persistência de antígenos bacterianos, que mantêm a resposta inflamatória por tempo prolongado. Motivo pelo qual o impacto da AS em termos de morbidade continua inalterada no decorrer dos anos, mesmo com o avanço medicamentos e tecnológico (ALVARES PA, et al., 2020; SOARES CBG, et al., 2019).

Assim, entendendo os danos que a AS pode trazer ao paciente com anemia falciforme (AF) e a necessidade de um cuidado cada vez mais eficaz e individualizado, a equipe de enfermagem juntamente com seus residentes, implementaram a SAE em seu processo de cuidado ao paciente deste relato de experiência, o que lhes permitiu delimitar com base no quadro clinico do paciente, os principais diagnósticos de enfermagem e as intervenções necessárias para seu reestabelecimento físico e biológico.

Dentre todos os diagnósticos de enfermagem (DE), tem-se o de "Risco de infecção", diagnóstico que segundo a literatura disponível relacionada a portadores de AF, é a complicação mais comum nestes pacientes, além do risco de choque séptico ou meningite por streptococcues pneumoniae ou haemophilus influenzae que aumentam o risco de morte principalmente em crianças falcêmicas. Situação que exige constante atenção da equipe de enfermagem, quanto a apresentação dos sinais vitais, com foco especial na temperatura corporal, e exames laboratoriais do paciente, tendo consideração que a febre atua como um forte sinal de infecção e potencial complicação (BRASIL, 2012).

Outro DE que podemos destacar é a "Dor aguda", sendo esta a apresentação mais comum das artrites sépticas, presente em $80 \%$ dos casos, podendo ou não está acompanhada de edema, redução da mobilidade e/ou febre (AKINKUGBE O, et al., 2019). Frente a este DE, o enfermeiro tem como premissa o controle das queixas álgicas, a fim de proporcionar maior conforto e bem estar ao paciente. Sendo para isso necessário o conhecimento e uso da escala da dor e realização de uma avaliação completa durante sua visita de enfermagem. Destaca-se que o conhecimento da fisiopatologia da dor, dos métodos farmacológicos e não farmacológicos de alívio, pode auxiliar o profissional de saúde na prevenção e identificação de crises álgicas, de forma a favorecer as ações de autocuidado e redução das complicações hemodinâmicas decorrentes do processo de dor (BRASIL, 2012).

Dentre as complicações hemodinâmicas evidenciadas em nosso relato de experiência, tem-se a troca de gases prejudicada, que se caracteriza por uma alteração na oxigenação nas membranas alvéolo-capilares, que pode ser evidenciada quando o paciente apresenta saturação menor que $95 \%$. Condição que necessita de intervenção imediata da equipe de saúde para resolução do quadro hipoxêmico e assim retorno do bom 
funcionamento cardíaco e respiratório do paciente. Para tanto o enfermeiro, como os demais profissionais, deve em sua metodologia de trabalho analisar os achados encontrados para determinar os diagnósticos de Enfermagem e suas respectivas intervenções (ROLIM ILTP, et. al., 2014).

Por fim, tem-se a redução do no nível de consciência, que é uma realidade comum em pacientes críticos com AF e AS, que configura um risco para aspiração de secreções na árvore traqueobrônquica, que, por conseguinte predispõe ao desenvolvimento de pneumonias aspirativas. Outros fatores de risco como a gastroparesia ocasionada pelo uso de opióides e presença de tudo endotraqueal, também podem colaborar para a ocorrência deste evento. Sendo de competência do profissional enfermeiro e fisioterapeuta a realização da técnica de aspiração das vias áreas superiores, como medida preventiva das complicações pulmonares, que aumentam o tempo de internação e mortalidade (AMARAL SM, et al., 2014).

Em suma, pode-se concluir que o processo de Enfermagem como caminho metodológico deve ser baseado nas melhores evidências científicas, uma vez que está fornece meios para o estabelecimento de condutas adequadas, direcionadas aos problemas de saúde, contribuindo para a recuperação e reabilitação do paciente. Ao paciente com hemoglobinopatia a implementação do processo de Enfermagem fundamentou a tomada de decisão na resolução da instabilidade hemodinâmica, melhora da oxigenação, conforto e redução de riscos relacionados a assistência à saúde.

\section{AGRADECIMENTOS}

Agradecemos a toda equipe multiprofissional da UTI, em especial, à equipe de enfermagem que nos auxiliou na construção de uma assistência adequada ao adolescente, de forma qualificada e humanizada.

\section{REFERÊNCIAS}

1. ADEGBOLA MA. Genomics and Pain Research in Sickle Cell Disease: An Explanation of Heterogeneity. ISRN Nursing, 2011; 1: 1-6.

2. AKINKUGBE O, STEWART C, MCKENNA C. Presentation and investigation of pediatric bone and joint infections in the pediatric emergency department. Pediatr Emerg Care, 2019; 35: 700-4.

3. ALVARES PA, MIMICA MJ. Osteoarticular infections in pediatrics. J Pediatr (Rio J). 2020; 96(S1): 58-64.

4. AMARAL SM, et al. Pneumonia nosocomial: importância do microambiente oral. J. Bras. Pneumol., $2014 ; 35$ (11): 1116-1124.

5. ASSUNÇÃO JH, et al. Artrite séptica do ombro e do cotovelo: análise epidemiológica de uma década em um hospital terciário. Revista Brasileira de Ortopedia, 2018; 53(6): 707-713.

6. BRASIL. Ministério da Saúde. Manual de Condutas básicas na doença falciforme. Secretaria de Atenção à Saúde. Departamento de Atenção Especializada, Brasília-DF. Ed. MS, 2012: 1-63.

7. CARVALHO EMMS. A pessoa com doença falciforme em uma unidade de emergência: limites e possibilidades para o cuidar da equipe de enfermagem. Dissertação (Mestrado) - Universidade Federal Fluminense, Niterói, $2014 ; 143$ p.

8. COTTLE RN, et al. Treating hemoglobinopathies using gene-correction approaches: promises and challenges. Hum Genet, 2016; 135 (9): 993-1010.

9. GUEDES A, DALTRO G. Infecções ósteo infecções ósteo-articulares na anemia f ticulares na anemia falciforme alciforme. Gaz. méd. Bahia 2010; 80:3(Ago-Out.): 85-88.

10. HABARA A, STEINBERG MH. Minireview: Genetic basis of heterogeneity and severity in sickle cell disease. Exp Biol Med, 2016; 241 (7): 689-96.

11. KENNEDY $\mathrm{N}$, et al. Native joint septic arthritis: epidemiology, clinical features, and microbiological causes in a New Zealand population. Journal of Rheumatology, 2015; 42 (12):2392-7

12. ROCHA LPO, et al. Assistência de enfermagem frente à problemática clínica de pacientes portadores de anemia falciforme. Revista Eletrônica Univar, 2014; 12 (2):44-48

13. RODRIGUES L. O papel do enfermeiro na unidade de terapia intensiva diante de novas tecnologias em saúde. Revista Saúde em Foco, 2018; 10: 412-428.

14. ROLIM ILTP, et al. O cuidado do paciente com troca de gases prejudicada utilizando as taxonomias de enfermagem - 61 CONGRESSO BRASILEIRO DE ENFERMAGEM 07 a 10 de dezembro de 2014.

15. SOARES CBG, et al. Infecção osteoarticular em crianças e adolescentes. Revista Mineira de Ortopedia e Traumatologia, 2019; 10(11):9-12.

16. SOARES EPB, et al. Cuidar de pessoas com doença falciforme na unidade de emergência: discurso de uma equipe multiprofissional. Revista Cienc cuid Saúde, 2014; 13 (2):278-285. 